\title{
FELIX ALEXANDROVICH BEREZIN AND HIS WORK
}

\author{
ALEXANDER KARABEGOV, YURI NERETIN, AND THEODORE VORONOV
}

To the memory of F. A. Berezin (1931-1980)

\begin{abstract}
This is a survey of Berezin's work focused on three topics: representation theory, general concept of quantization, and supermathematics.
\end{abstract}

\section{Preface}

This text has resulted from our participation in the XXXth Workshop on Geometric Methods in Physics held in Białowieża in summer 2011. Part of this conference was a special Berezin Memorial Session: Representations, Quantization and Supergeometry. F. A. Berezin, who died untimely in 1980 in a water accident during a trip to Kolyma, would have been eighty in 2011.

This is an attempt to give a survey of Berezin's remarkable work and its influence for today. Obviously, we could not cover everything. This survey concentrates on three topics: representation theory, quantization and supermathematics. Outside of its scope remained, in particular, some physical works in which Berezin was applying his approach to second quantization and his theory of quantization. Also, we did not consider two important but somewhat stand-alone topics of the latest period of Berezin's work devoted to an interpretation of equations such as KdV from the viewpoint of infinite-dimensional groups [49, 50] (joint with A. M. Perelomov) and a method of computing characteristic classes 53 (joint with V. S. Retakh).

For a sketch of Berezin's life and personality, we refer to a brilliant text by R. A. Minlos 93 .

Sections 2 and 3 below were written by Yu. A. Neretin. Section 4 was written by A. V. Karabegov. Section 5 was written by Th. Th. Voronov, who also proposed the general plan of the paper and made the final editing.

\section{LAPLACE OPERATORS ON SEMISIMPLE LIE GROUPS}

The main scientific activity of F. A. Berezin was related with mathematical physics, quantization, infinite-dimensional analysis and infinite-dimensional groups, and supermathematics. But in 1950s he started in classical representation theory (which at that time was new and not yet classical).

Date: 16 (29) February 2012.

1991 Mathematics Subject Classification. Primary 01A70; Secondary 58A50, 58Z99, 22E66, $81 \mathrm{~S} 99$.

Key words and phrases. Representation theory, Laplace operators, second quantization, deformation quantization, symbols, supermanifolds, Lie supergroups. 
2.1. Berezin's Ph.D. thesis: characters of complex semisimple Lie groups and classification of irreducible representations. Our first topid 1 is the cluster of papers 1956-57: announcements [7, 8], 40, [9, the main text [10, and an addition in [16. This work has a substantial overlap with Harish-Chandra's papers of the same years, see [76. F. A. Berezin in 1956 claimed that he classified all irreducible representations of complex semisimple Lie groups in Banach spaces. We shall say a few words about this result and the approach, which is interesting no less than the classification.

The technology for construction representations of semisimple groups (parabolic induction and principal series) was proposed by I. M. Gelfand and M. A. Naimark in book [0]. On the other hand, Harish-Chandra 75] in 1953 proved the 'subquotient theorem': each irreducible representation is a subquotient of a representation of the principal (generally, non-unitary) series.

Consider a complex semisimple (or reductive) Lie group $G$, its maximal compact subgroup $K$ and the symmetric space $G / K$. For instance, consider $G=\operatorname{GL}(n, \mathbb{C})$; then $K=\mathrm{U}(n)$ and $G / K$ is the space of positive definite matrices of order $n$. A Laplace operator is a $G$-invariant partial differential operator on $G / K$. Let us restrict a Laplace operator to the space of $K$-invariant functions (for instance, in the example above it is the space of functions depending on eigenvalues of matrices). The radial part of Laplace operator is such a restriction.

Berezin described explicitly the radial parts of the Laplace operators on $G / K$. He showed that in appropriate coordinates $t_{1}, \ldots, t_{n}$ on $K \backslash G / K$ each radial part has the form

$$
p\left(\frac{\partial}{\partial t_{1}}, \ldots \frac{\partial}{\partial t_{n}}\right)
$$

where $p$ is a symmetric (with respect to the Weyl group) polynomial.

The first application was a proof of the formula for spherical functions on complex semisimple Lie groups from Gelfand and Naimark's book 70. One of the possible definitions of spherical functions: they are $K$-invariant functions on $G / K$ that are joint eigenfunctions for the Laplace operators. I. M. Gelfand and M. A. Naimark proved that for $G=\mathrm{GL}(n, \mathbb{C})$ such functions can be written in the terms of the eigenvalues $e^{t_{k}}$ as

$$
\Phi_{\lambda}(t)=\operatorname{const}(\lambda) \cdot \frac{\operatorname{det}_{k, m}\left\{e^{\lambda_{k} t_{m}}\right\}}{\operatorname{det}_{k, m}\left\{e^{k t_{m}}\right\}}
$$

as in the Weyl character formula for finite-dimensional representations of $\operatorname{GL}(n, \mathbb{C})$, but the exponents $\lambda_{j}$ are complex. They wrote the same formula for other complex classical groups, but it seems that their published calculation 3 can be applied only for $\operatorname{GL}(n, \mathbb{C})$. Berezin reduced the problem to a search of common eigenvalues of operators (11) and solved it!

Next, consider Laplace operators on a complex semisimple Lie group $G$, i.e. differential operators invariant with respect to left and right translations on $G$. We

\footnotetext{
${ }^{1}$ This was not the first work of Berezin. The paper [39] of Berezin and I. M. Gelfand (1956) on convolution hypergroups was one of the first attacks on the Horn problem; in particular they showed a link between eigenvalue inequalities and tensor products of irreducible representations of semisimple groups, see 86, 69.

${ }^{2}$ We also allow change $f(t) \mapsto \alpha(t) f(t)$.

${ }^{3}$ It is very interesting, an integration in the Jacobi elliptic coordinates.

${ }^{4}$ The function $\alpha$ from a previous footnote is the denominator of (2).
} 
can consider $G$ as a symmetric space, it acts on itself by left and right translations, $g \mapsto h_{1}^{-1} g h_{2}$, the stabilizer of the point $1 \in G$ is the $\operatorname{diagonal} \operatorname{diag}(G) \subset G \times G$, i.e., we get the homogeneous space $G \times G / \operatorname{diag}(G)$. Note also that $G \times G / \operatorname{diag}(G)$ is the complexification of the space $G / K$. We again can consider the radial parts of Laplace operators as the restrictions of Laplace operators to the space of functions depending on eigenvalues $\lambda_{j}$. Since now eigenvalues are complex, the formula transforms to

$$
p\left(\frac{\partial}{\partial t_{1}}, \ldots \frac{\partial}{\partial t_{n}} ; \frac{\partial}{\partial \bar{t}_{1}}, \ldots \frac{\partial}{\partial \bar{t}_{n}}\right),
$$

where $p$ is separately symmetric with respect to holomorphic and anti-holomorphic partial derivatives 5 .

Recall that for infinite-dimensional representations $\rho$ the usual definition of the character $\chi(g)=\operatorname{tr} \rho(g)$ makes no sense, because an invertible operator has no trace. However, for irreducible representations of semisimple Lie groups and smooth functions $f$ with compact supports the operators $\rho(f)=\int f(g) \rho(g)$ are of trace class. Therefore $f \mapsto \operatorname{tr} f(g)$ is a distribution on the group in the sense of L. Schwartz. This is the definition of the character of an irreducible representation.

A character is invariant with respect to the conjugations $g \mapsto h g h^{-1}$. Also, it is easy to show that a character is an eigenfunction of all Laplace operators. The radial parts of Laplace operators were evaluated, so we can look for characters as joint eigenfunctions of operators (3). Algebraically the problem is similar to calculation of spherical functions and final formulas are also similar (but there are various additional analytic difficulties).

For a generic eigenvalue, a symmetric solution is unique. It has the form

$$
\sum_{\sigma \in S_{n}}(-1)^{\sigma} e^{\sum_{k}\left(p_{k} t_{\sigma(k)}+q_{k} \bar{t}_{\sigma(k)}\right)},
$$

for $G=\mathrm{GL}(n, \mathbb{C})$, here $S_{n}$ is the symmetric group. This is the character of a representation of the principal series. For 'degenerate' cases there are finite subspaces of solutions. Berezin showed that all characters are linear combinations of the characters of representations of principal series. In the introduction to [10], he announced without proof a classification of all irreducible representations. The restriction of a representation of the principal series to $K$ contains a unique subrepresentation with the minimal possible highest weight6. We must choose a unique subquotient containing this representation of $K$.

A formal proof of the classification of representations was not presented in 10 , but the theorem about characters and the classification theorem are equivalent 7 .

Paper [10] was written in an enthusiastic style and was not always careful. J. M. G. Fell, Harish-Chandra, A. A. Kirillov, and G. M. Mackey formulated two critical arguments; Berezin responded in a separate paper [16].

\footnotetext{
${ }^{5}$ The eigenfunctions of (3) are exponential and we have to symmetrize them because we need symmetric solutions.

${ }^{6}$ In 1966 D. P. Zhelobenko and M. A. Naimark 127 announced the classification theorem in a stronger form. Later (1967-1973) D. P. Zhelobenko published a series of papers on complex semisimple Lie groups, e.g. 126, where he, in particular, presented a proof of this theorem (with a contribution of M. Duflo).

${ }^{7}$ It is not difficult to show that the distinct subquotients have different characters. The transition matrix between the characters of the principal series and the characters of irreducible representations is triangular with units on the diagonal.
} 
Firstly, the original Berezin work contains a non-obvious and unproved lemma (on the correspondence between solutions of the systems of PDE in distributions on the group and the system of PDE in radial coordinates). A proof was a subject of the additional paper [16.

Secondly, Berezin actually worked with irreducible representations whose $K$-spectra have finite multiplicities (i.e, the irreducible Harish-Chandra modules). He formulated the final result as the 'classification of all irreducible representations in Banach spaces' and at this point he claimed that the equivalence of the two concepts had been proved by Harish-Chandra. But this is not correct8. He had to formulate the statement as the "classification of all completely irreducibl@9 representations in Banach spaces", with the necessary implication proved by R. Godement [71] in 1952.

Recall that the stronger version of classification theorem was proved by Zhelobenko near 1970. For real semisimple groups, the classification was announced by R. Langlands in 1973 and proofs were published by A. Borel and N. Wallach in 1980 .

2.2. Radial parts of Laplace operators. Spherical functions, the spherical transform, and the radial parts of Laplace operators appeared in representation theory in the 1950s. Later they became important in integrable systems. On the other hand, they gave a new start for the theory of multivariable special functions (I. G. Macdonald, H. Heckman, E. Opdam, T. Koornwinder, I. Cherednik, and others.).

Consider a real semisimple Lie group $G$, its maximal compact subgroup $K$ and the Riemannian symmetric space $G / K$. If the group $G$ is complex, then the spherical functions are elementary functions, as we have seen above.

But for the simplest of the real groups, $G=\mathrm{SL}(2, \mathbb{R})$, the spherical functions are the Legendre functions. In this case, the radial part of the Laplace operator is a hypergeometric differential operator (with some special values of the parameters). General spherical functions are higher analogs of the Gauss hypergeometric functions. Respectively, the radial parts of the Laplace operators are higher analogs of hypergeometric operators (see expressions in 112 and 77, Chapter 1). The first attack in this direction was made by F. A. Berezin and F. I. Karpelevich 44 in 1958.

Berezin and Karpelevich found a semi-elementary case, the pseudounitary group $G=\mathrm{U}(p, q)$. In this case the radial parts of Laplace operators are also symmetric expressions of the form

$$
r\left(L\left(x_{1}\right), \ldots, L\left(x_{p}\right)\right)
$$

but $L(x)$ is now a second order (hypergeometric) differential operator,

$$
D:=x(x+1) \frac{d^{2}}{d x^{2}}+[(q-p+1)+(q-p) x] \frac{d}{d x}+\frac{1}{4}(q-p+1)^{2} .
$$

They also evaluated the spherical functions on $\mathrm{U}(p, q)$ as eigenfunctions of the radial Laplace operators. In appropriate coordinates the functions have the form

$$
\Phi_{s}(x)=\mathrm{const} \cdot \frac{\operatorname{det}_{k, j}\left\{{ } _ { 2 } F _ { 1 } \left[\begin{array}{c}
\left.\left.\frac{1}{2}(q-p+1)+i s_{j}, \frac{1}{2}(q-p+1)-i s_{j} ;-x_{k}\right]\right\} \\
q-p+1
\end{array} ;\right.\right.}{\prod_{1 \leq k<l \leq p}\left(s_{k}^{2}-s_{l}^{2}\right) \prod_{1 \leq k<l \leq p}\left(x_{k}-x_{l}\right)} .
$$

\footnotetext{
${ }^{8}$ These two properties are not equivalent, see Soergel's counterexample [117.

${ }^{9}$ There are many versions of irreducibility for infinite-dimensional non-unitary representations. A representation is completely irreducible if the image of the group algebra is weakly dense in the algebra of all operators.
} 
Here ${ }_{2} F_{1}[\ldots]$ is the Gauss hypergeometric function, $x_{1}, \ldots, x_{p}$ are coordinates on the Cartan subgroup of $\mathrm{U}(p, q)$, and $s_{1}, \ldots, s_{p}$ are parameters of spherical functions.

This paper was accepted by Doklady in June 1957. Near that time Berezin's scientific interests had changed and he left the classical representation theory 10.11 .

(The next step was done by M. A. Olshanetsky and A. M. Perelomov [101] in 1976; see also 102. They wrote the radial part of the second order Laplace operator. Quite soon J. Sekiguchi [112] obtained a general formula for the groups GL.)

\section{Method of SECOND QUANTIZATion}

Our next topic is the famous book "The method of second quantization" 14] (and the announcements [11, 48, 12, 13]). A more detailed discussion of the intellectual history of this work and its influence is in 98 .

3.1. Prehistory. It is known that at the end of 1950s Berezin started to learn physics and to participate in theoretical physics seminars in Moscow. He had to decide between numerous possible ways in this new world and his choice was the problem about the automorphisms of the canonical commutation and anticommutation relations formulated in the book 'Mathematical aspects of the quantum theory of fields' by K. O. Friedrichs [68] of 1953.

Let $P_{1}, \ldots, P_{n}, Q_{1}, \ldots, Q_{n}$ be self-adjoint operators in a Hilbert space satisfying the conditions

$$
\left[P_{k}, P_{l}\right]=\left[Q_{k}, Q_{l}\right]=0, \quad\left[P_{k}, Q_{l}\right]=i \delta_{k, l}
$$

and without a common invariant subspace. Such conditions are called the canonical commutation relations, abbreviation CCR. According to the Stone-von Neumann theorem, such a system of operators is unique up to a unitary equivalence (for a precise forms of the theorem, see, e.g., [15]). In fact, our Hilbert space can be identified with $L^{2}\left(\mathbb{R}^{n}\right)$ and the operators with $x_{k}, i \frac{\partial}{\partial x_{k}}$, respectively. Now let $g=\left(\begin{array}{ll}\alpha & \beta \\ \gamma & \delta\end{array}\right)$ be a symplectic $2 n \times 2 n$ matrix. Evidently, the operators

$$
P_{k}^{\prime}=\sum_{l} \alpha_{k l} P_{l}+\sum_{l} \beta_{k l} Q_{k l}, \quad Q_{k}^{\prime}=\sum_{l} \gamma_{k l} P_{l}+\sum_{l} \delta_{k l} Q_{k l}
$$

satisfy the same relations (4). Therefore there is a unitary operator $U=U(g)$ such that 12

$$
P_{k}^{\prime}=U(g) P_{k} U(g)^{-1}, \quad Q_{k}^{\prime}=U(g) Q_{k} U(g)^{-1} .
$$

\footnotetext{
${ }^{10}$ In 1976 paper 27] and the five ITEP preprints of 1977 included in the English version of 38, Berezin returned to the study of Laplace operators and considered the radial parts of Laplace operators for Lie supergroups (see subsection 5.5). They are usual (non-super) partial differential operators. This topic is not well understood up to now; A. N. Sergeev and A. P. Veselov produced from this standpoint new operators of Calogero-Moser type whose eigenfunctions are super-Jack functions (which also are functions of even variables), see [113. On an analog of the group case, see 78 .

${ }^{11}$ In 1970s, Berezin made a work on the harmonic analysis in Hilbert spaces of holomorphic functions [23], 26], 34] for a discussion of this work and its continuations, see [118, 97, and [99], Chapter 7 .

${ }^{12}$ Now the mapping $g \mapsto U(g)$ is called the Weil representation, see A. Weil's paper [125], 1964 . The term is common and convenient, but historically it was a construction due to K. O. Friedrichs and I. Segal.
} 
By a version of the Schur Lemma, this operator is unique up to a scalar factor. It is easy to see that

$$
U(g) U(h)=\lambda(g, h) U(g, h),
$$

where $\lambda(\cdot, \cdot)$ is a complex scalar. Apparently, Friedrichs decided that there was nothing to discuss here and asked what would happen if the number $n$ of the operators were $\infty$. He showed that there are many nonequivalent representations of CCR besides the well-known Fock representation. Next, Friedrichs asked, for which symplectic matrices the system of operators $P_{k}^{\prime}, Q_{k}^{\prime}$ are equivalent to $P_{k}$, $Q_{k}$. He formulated a correct conjecture and tried to find explicit formulas for $U(g)$.

3.2. Operators and divergences. Consider the usual Fourier transform $\mathcal{F}$ in $L^{2}(\mathbb{R}), \widehat{f}(\xi)=\int e^{i x \xi} f(x) d x$. Its definition is not completely straightforward, since the integral can be divergent, and some regularization dance is necessary. If we want to find $\mathcal{F}^{2}$, we must calculate the kernel

$$
K(x, y)=\int e^{i y \xi} e^{i x \xi} d \xi
$$

Since we know the answer, we can believe that it is obvious. In any case, the integral diverges...

These difficulties are usual for the work with integral operators in $L^{2}\left(\mathbb{R}^{n}\right)$. Field theory requires functions of infinite number of variables and passing to the limit $n \rightarrow \infty$ only multiplies the problems. Berezin noticed that in the space $F_{n}$ of entire functions on $\mathbb{C}^{n}$ with the inner product

$$
\langle f, g\rangle=\frac{1}{(2 \pi)^{n}} \int f(z) \overline{g(z)} e^{-|z|^{2}} d \Re(z) d \Im(z)
$$

we can realize our operators as

$$
P_{k}=\frac{1}{\sqrt{2}}\left(z_{k}+\frac{\partial}{\partial z_{k}}\right), \quad Q_{k}=\frac{1}{\sqrt{2} i}\left(z_{k}-\frac{\partial}{\partial z_{k}}\right) .
$$

Therefore this space can be identified with $L^{2}\left(\mathbb{R}^{n}\right)$. Berezin observed that in the space $F_{n}$ any bounded operator is an integral operator of the form

$$
A f(z)=\int_{\mathbb{C}^{n}} K(z, \bar{u}) f(u) e^{-|u|^{2}} d u d \bar{u}
$$

and the integral is convergent. Also the kernel of a product of integral operators is defined by a convergent integral. Next, Berezin showed that this 'holomorphic model' perfectly survives as $n \rightarrow \infty$ (only the case $n=\infty$ is discussed in book [14, Berezin uses the term 'generating functional' for the function assigned to an operator). In particular, we can work with bounded operators without any divergences.

Certainly, we need also unbounded operators, where divergent expressions have to appear. But, again, the 'level of divergences' is minimal.

In parallel, Berezin proposed an almost equivalent formalism of Wick symbols. Algebraically, they looks similar to the well-known since 1930s expressions of operators as $A=p\left(x, \frac{\partial}{\partial x}\right)$, where all $x$ 'a are at the left and all $\frac{\partial}{\partial x}$ 's are at the right. But only few operators can be written in this form if we understand 'functions' literally. In contrast, we can express an operator as $A=p\left(z, \frac{\partial}{\partial z}\right)$ more or less always. 
3.3. Weil representation. Using this operator formalism, Berezin wrote explicit formulas for the operators $U(g)$. He interpreted the conditions (66) as a first order system of PDEs for the kernels $K$ of $U(\cdot)$, solved the equations and got the expressions of the form

$$
K(z, \bar{u})=\exp \{S(z, \bar{u})\}
$$

where $S$ is an explicit quadratic form. Thus we obtain a projective representation of an infinite-dimensional symplectic group by integral operators acting in the space of functions of infinite number of variables. We also can replace $\infty \mapsto n$ and obtain a construction that was completely new in that time.

In particular, Berezin proved the Friedrichs conjecture about the domain of definition of this representation.

3.4. Fermionic Fock space. For us a fermionic Fock space is a space of functions of anticommuting variables. This idea, now common, originated from Berezin's book [14. Berezin also found that there is a natural integral over anticommuting variables ([11). We say more about that in section 5. Berezin showed that an operator in the fermionic Fock space is determined by a function (the 'generating functional') depending on a double collection of anticommuting variables and that it is convenient to express operators in a fermionic Fock space as integral operators, with respect to that peculiar integral.

In 68, Friedrichs also formulated a problem about the canonical anticommutation relations (abbreviation CAR)

$$
\left\{P_{k}, P_{l}\right\}=\left\{Q_{k}, Q_{l}\right\}=0, \quad\left\{P_{k}, Q_{l}\right\}=i \delta_{k, l}
$$

and their symmetries (5). Now the matrix $g=\left(\begin{array}{ll}\alpha & \beta \\ \gamma & \delta\end{array}\right)$ is orthogonal. Berezin solved this problem as well and wrote a formula for the kernels of $U(\cdot)$,

$$
K(\xi, \eta)=\exp \{S(\xi, \eta)\},
$$

where $S$ is an explicit quadratic expression. Note that the formulas for $S$ in (7) and (9) are similar.

In fact, the both theorems are results in the representation theory of infinitedimensional Lie groups. Berezin's book can be regarded as a mathematization of field theory. However, it was also (Chapters 2 and 3) the first book on infinitedimensional groups and the start of this theory. For a more detailed discussion, see 98 .

3.5. History and references. Main Berezin's results with outlined proofs were announced in Doklady paper [1], of March 1961 (accepted in November 1960). The text was written in the telegraphic style usual for Doklady of that time: the allowed four pages were all used up to one line. In September 1962, Berezin submitted a large paper to Uspehi (that is, Russian Mathematical Surveys). The paper was rejected. In the following years, Berezin published more short announcements: [12], 13 and 48 . In 1965 13 the book "The method of second quantization" was published, addressed to physicist:14.

\footnotetext{
${ }^{13}$ The English version appeared in 1966.

${ }^{14}$ In spite of its physical language, the book is a rigorous, maybe not detailed, mathematical text.
} 
Friedrichs's questions also attracted Irving Segal, who had worked in mathematical field theory since the beginning of 1950s. (In particular, Segal introduced a model of the Fock space as $L^{2}$ on a Gaussian measure [110], 1956; later J. Feldman [67, 1959, constructed the action of an infinite-dimensional GL on that space.) In 1959, Segal obtained explicit formulas for the 'Weil representation' for finite $n$ in the space $L^{2}$, 111. In 1961, he proposed a holomorphic model for the boson Fock space (this was also done by V. Bargmann 2 in the same year). In 1962, D. Shale [114] published the solution of the Friedrichs problem for CCR, and in 1965, D. Shale and R. W. Stinespring published their solution for CAR 115 .

However these papers did not cover Berezin's results. His book and Berezin himself immediately became famous.

3.6. Berezin's book in physics. Besides the formal results concerning CCR and CAR, the interest of physicists to this text had two additional reasons.

First, the new operator formalism (both bosonic and fermionic) was very convenient. It became easier to write formulas and to calculate.

The second reason was the mysterious parallelism between the bosonic and fermionic spaces which was emphasized in the book. For Berezin himself this was the starting point of his work leading to the creation of supermathematics (see section 5).

\section{BEREZIN'S GENERAL CONCEPT OF QUANTIZATION}

One of the main directions of Berezin's research was mathematical formulation of the concept of quantization as a deformation of a classical mechanical system. In [18 Berezin interpreted the universal enveloping algebra of a Lie algebra as a quantization of the Poisson algebra of polynomial functions on the dual of the Lie algebra. In 24] and 25] Berezin introduced a general concept of quantization based upon algebras of operator symbols depending on a small parameter.

Quoting from [59], "according to the main idea of these works, quantization has the following precise mathematical meaning: the algebra of quantum observables is a deformation of the algebra of classical observables, so that the Planck constant plays the role of the deformation parameter and the direction of deformation (the first derivative in the parameter at zero) is given by the Poisson bracket"

In [26], Berezin studied quantization of complex symmetric spaces. The operator symbols used in quantization were introduced and studied in [20, 21], and [22]. In 34, Berezin obtained the spectral decomposition of the operator connecting covariant and contravariant symbols on classical complex symmetric spaces, now called the Berezin transform. In [19] and 37, Berezin constructed finite approximations of Feynman path integrals with the use of operator symbols. See also Berezin and M. A. Shubin [54 and their joint book "The Schrödinger Equation" [55], which Shubin prepared for publication after Berezin's death.

Let us consider these works in greater detail.

4.1. Poisson bracket and quantization on the dual of a Lie algebra. In the fundamental paper [18] Berezin constructed an integral transform $\delta$ from generalized functions on a neighborhood of the identity of a Lie group $G$ to functions on the dual $\tilde{\mathfrak{G}}$ of its Lie algebra $\mathfrak{G}$ and expressed the symmetrization mapping $\Lambda$ from

\footnotetext{
${ }^{15}$ Note that the famous mathematicians D. Shale, R. W. Stenespring and J. Feldman were all I. Segal's students.
} 
the symmetric algebra $S$ of $\mathfrak{G}$ to the universal enveloping algebra $\hat{S}$ of $\mathfrak{G}$ through the mapping $\delta$. Let $\left\{\hat{x}_{p}\right\}$ be a basis in $\mathfrak{G},\left\{t_{p}\right\}$ the corresponding coordinates on $\mathfrak{G}$, $\left\{y_{p}\right\}$ the dual coordinates on $\tilde{\mathfrak{G}}$. The mapping $\delta$ is defined as follows:

$$
\delta: s(g) \mapsto \stackrel{\delta}{s}(y)=\int e^{-i t y} s(g(t)) \rho(t) d t, \quad t y=\sum_{p} t_{p} y_{p},
$$

where $t \mapsto g(t)$ is the exponential mapping and $\rho(t)$ is the density of the rightinvariant measure on $G$ in the canonical coordinates $\left\{t_{p}\right\}$. The symmetric algebra $S$ of $\mathfrak{G}$ is identified with the space of polynomials on $\tilde{\mathfrak{G}}$ and $\Lambda$ maps $y_{p}$ to $\hat{y}_{p}=-i \hat{x}_{p}$. The generalized functions supported at the identity of $G$ form an algebra with respect to the convolution. This algebra is naturally identified with the universal enveloping algebra $\hat{S}$. Berezin proved that under this identification the inverse mapping $\Lambda^{-1}$ is given by the mapping $\delta$. The mapping $\delta$ allows to transfer the convolution of generalized functions supported on a small neighborhood $U$ of the identity of the group $G$ to an operation on functions on the dual $\tilde{\mathfrak{G}}$ of the Lie algebra $\mathfrak{G}$. Berezin gave an integral formula for this operation. Given generalized functions $s_{1}, s_{2}$ supported on $U$ and their convolution $s$, set $\sigma_{1}=\stackrel{\delta}{s}, \sigma_{1}=\stackrel{\delta}{=}$ and $\sigma=\stackrel{\delta}{s}$. Then

$$
\sigma(y)=\int K_{U}\left(y \mid y_{1}, y_{2}\right) \sigma_{1}\left(y_{1}\right) \sigma_{2}\left(y_{2}\right) d y_{1} d y_{2},
$$

where

$$
K_{U}\left(y \mid y_{1}, y_{2}\right)=\frac{1}{(2 \pi)^{2 n}} \int_{g\left(t_{1}\right) \in U, g\left(t_{2}\right) \in U} e^{-i y \log \left(g\left(t_{1}\right) g\left(t_{2}\right)\right)+i y_{1} t_{1}+i y_{2} t_{2}} d t_{1} d t_{2} .
$$

Berezin noted that this integral formula can be extended to the space $S$ of polynomials on $\tilde{\mathfrak{G}}$ and the resulting algebra is isomorphic to the universal enveloping algebra $\hat{S}$ of $\mathfrak{G}$. Moreover, the leading term of the commutator of polynomials leads to a natural Poisson bracket on the dual of the Lie algebra $\mathfrak{G}$. For arbitrary smooth functions on $\tilde{\mathfrak{G}}$ it is possible to write

$$
\left\{f_{1}, f_{2}\right\}=\sum C_{i j}^{k} y_{k} \frac{\partial f_{1}}{\partial y_{i}} \frac{\partial f_{2}}{\partial y_{j}}
$$

where $C_{i j}^{k}$ are the structure constants of the Lie algebra $\mathfrak{G}$. About the same time, this Poisson bracket on $\tilde{\mathfrak{G}}$ (in the form of a symplectic structure on the coadjoint orbits) was discovered in the orbit method. Therefore it became known as the Berezin-Kirillov or Berezin-Kirillov-Kostant bracket. (Later Alan Weinstein found out that the bracket had been known already to S. Lie, so the name 'LiePoisson bracket' became more standard.)

Thus the universal enveloping algebra of $\mathfrak{G}$ can be interpreted as a quantization of the corresponding Poisson algebra on $\tilde{\mathfrak{G}}$ consisting of the polynomial functions endowed with the Berezin-Kirillov-Kostant Poisson bracket.

In fact, by rescaling this operation by a formal parameter $\hbar$, one can obtain from above Berezin's formula the following integral formula for what is now known as the 'BakerCampbell-Hausdorff star product' on $\tilde{\mathfrak{G}}$ :

$$
\begin{aligned}
& \left(f_{1} * f_{2}\right)(y)= \\
& \quad \frac{1}{(2 \pi \hbar)^{n}} \iint d y_{1} d t_{1} d y_{2} d t_{2} f_{1}\left(y_{1}\right) f_{2}\left(y_{2}\right) e^{-\frac{i}{\hbar}\left(\left\langle t_{1}, y_{1}\right\rangle+\left\langle t_{2}, y_{2}\right\rangle-\left\langle H\left(t_{1}, t_{2}\right), y\right\rangle\right)} .
\end{aligned}
$$


Here $H\left(t_{1}, t_{2}\right)$ is the formal $\mathrm{BCH}$ power series on $\mathfrak{G}$ and the integration extends over $\tilde{\mathfrak{G}} \times \mathfrak{G} \times \tilde{\mathfrak{G}} \times \mathfrak{G}$. The functions $f_{1}$ and $f_{2}$ can be arbitrary smooth functions on $\tilde{\mathfrak{G}}$ due to the presence of a formal parameter $\hbar 16$.

4.2. General concept of quantization as deformation. In [24] and 25] Berezin gave a general definition of quantization of a Poisson manifold $(M,\{\cdot, \cdot\})$ as an algebra $(\mathfrak{A}, *)$ of sections of a field of noncommutative algebras $\left(\mathcal{A}_{h}, *_{h}\right)$ parameterized by the elements $h$ of a set $E$ of positive numbers that has zero as an accumulation point. The Correspondence Principle for this quantization is expressed in terms of a homomorphism

$$
\varphi_{0}: \mathfrak{A} \rightarrow C^{\infty}(M)
$$

such that for $f, g \in \mathfrak{A}$,

$$
\varphi_{0}\left(\frac{1}{h}(f * g-g * f)\right)=i\left\{\varphi_{0}(f), \varphi_{0}(g)\right\} .
$$

Then he considered a special case when $\mathcal{A}_{h} \subset C^{\infty}(M)$, the elements of $\mathfrak{A}$ are functions $f(h, x)$ on $E \times M$, and

$$
\varphi_{0}(f)=\lim _{h \rightarrow 0} f(h, x) .
$$

4.3. Berezin's quantization using symbols. Berezin studied a number of examples of such special quantizations where $\mathcal{A}_{h}$ for a fixed $h$ is an algebra of symbols of operators in a Hilbert space. To this end, Berezin introduced covariant and contravariant symbols related to an overcomplete family of vectors in a reproducing kernel space. Namely, consider a Hilbert space $H$ and a set $M$ with measure $d \alpha$ whose elements parameterize a system of vectors $\left\{e_{\alpha}\right\}$ in $H$. Let $P_{\alpha}$ be the orthogonal projection operator onto $e_{\alpha}$ and

$$
d \mu(\alpha)=\left\|e_{\alpha}\right\|^{2} d \alpha
$$

be another measure on $M$. The vectors $\left\{e_{\alpha}\right\}$ form an overcomplete family in $H$ if

$$
\int P_{\alpha} d \mu(\alpha)=E
$$

is the identity operator in $H$. Then $H$ is isometrically embedded into $L^{2}(M, d \alpha)$ by the mapping $H \ni f \mapsto\left\langle f, e_{\alpha}\right\rangle$. The projectors $P_{\alpha}$ are used to define covariant and contravariant symbols of operators in $H$ as follows. The covariant symbol of an operator $\hat{A}$ is the function

$$
A(\alpha)=\operatorname{tr} \hat{A} P_{\alpha}
$$

on $M$. A function $\stackrel{\circ}{\mathrm{A}}(\alpha)$ on $M$ is a contravariant symbol of $\hat{A}$ if

$$
\hat{A}=\int P_{\alpha} \stackrel{\circ}{\AA}(\alpha) d \mu(\alpha) .
$$

The measure $\mu$ defines a trace functional on appropriate classes of covariant and contravariants symbols that agrees with the operator trace (see [21]),

$$
\operatorname{tr} \hat{A}=\int A d \mu=\int \stackrel{\circ}{\mathrm{A}} d \mu .
$$

\footnotetext{
${ }^{16}$ We obtained formula (10) around 1998 (Th.V., unpublished) and then realized that it can be deduced from Berezin 18 .
} 
The covariant and contravariant symbols $A$ and $\stackrel{\circ}{\mathrm{A}}$ of the same operator $\hat{A}$ are connected via the Berezin transform $I$,

$$
A(\alpha)=(I \stackrel{\circ}{\mathrm{A}})(\alpha)=\int \operatorname{tr}\left(P_{\alpha} P_{\beta}\right) \stackrel{\circ}{\mathrm{A}}(\beta) d \mu(\beta) .
$$

An overcomplete system of vectors $\left\{e_{\alpha}\right\}$ in $H$ may admit a symmetry group $G$ that acts upon $H$ by a unitary representation $g \mapsto U_{g}$ and upon $M$ by transformations preserving the equivalence class of the measure $d \alpha$ so that

$$
U_{g} e_{\alpha}=s(\alpha, g) e_{g \alpha},
$$

where $s: M \times G \rightarrow \mathbb{C}$ is a measurable cocycle satisfying

$$
\frac{d g \alpha}{d \alpha}=|s(\alpha, g)|^{2} \text {. }
$$

Then $U_{g} P_{\alpha} U_{g}^{-1}=P_{g \alpha}$, the measure $d \mu$ is $G$-invariant, the symbol mappings

$$
\hat{A} \mapsto \operatorname{tr} \hat{A} P_{\alpha} \text { and } \stackrel{\circ}{\mathrm{A}}(\alpha) \mapsto \int P_{\alpha} \stackrel{\circ}{\AA}(\alpha) d \mu(\alpha)
$$

are $G$-equivariant and the Berezin transform $I$ is $G$-invariant.

Berezin studied spectral properties of covariant and contravariant symbols in [21] and then used algebras of covariant symbols to define a quantization of a special class of Kähler manifolds in 24 using the saddle-point method. He started with a Kähler manifold $M$ of complex dimension $m$ with a Kähler form $\omega$ and the Liouville measure $\omega^{m}$. He assumed that there exists a global Kähler potential $\Phi$ of the form $\omega$ and introduced an $h$-parameterized family of measures

$$
d \alpha_{h}=\mathrm{e}^{-\frac{1}{h} \Phi} \omega^{m}
$$

on $M$. Then he considered the Hilbert space $H_{h}$ of holomorphic functions on $M$ square integrable with respect to the measure $d \alpha_{h}$. The Bergman reproducing kernel of $H_{h}$ defines an overcomplete system of vectors $\left\{e_{\alpha}^{(h)}\right\}$ in $H_{h}$. In order to prove the Correspondence Principle, Berezin imposed a severe assumption on $M$ that

$$
e_{\alpha}^{(h)}(z)=c(h) \mathrm{e}^{\frac{1}{h} \Phi(z, \bar{w})}
$$

for $\alpha=(w, \bar{w}) \in M$ and some constant $c(h)$. This assumption is satisfied on Kähler manifolds with a transitive symmetry group which allowed Berezin to quantize complex symmetric spaces (see [26]).

4.4. Influence of Berezin's work. In the following decades Berezin's work on quantization attracted a lot of attention. His results were expanded and generalized by many mathematicians and mathematical physicists in two major directions. First, Berezin's definition of quantization in the special case when $\mathcal{A}_{h} \subset C^{\infty}(M)$ was extended to incorporate deformation quantization of Flato et al. [6] as a formal asymptotic expansion in $h$ of the product $*_{h}$ in the algebra $\mathcal{A}_{h}$. In the general case this can be achieved by extending the homomorphism (11) in the general definition of quantization to a homomorphism

$$
\varphi=\varphi_{0}+\nu \varphi_{1}+\ldots: \mathfrak{A} \rightarrow C^{\infty}(M)[[\nu]]
$$

to the star-algebra of some formal deformation quantization on the Poisson manifold $(M,\{\cdot, \cdot\})$ such that $\varphi(h f)=\nu \varphi(f)$. Examples of such quantizations of

\footnotetext{
${ }^{17}$ Here $\nu$ is a formal parameter.
} 
Kähler manifolds were first given in 96 and 63,64 . The second direction was to remove the restrictions on the Kähler manifold in Berezin's quantization. Based on the microlocal technique developed by Boutet de Monvel and Guliiemin in 62], it was shown in 60, that Berezin-Toeplitz quantization 18 on general compact Kähler manifolds satisfies an analog of the Correspondence Principle. Then in 106 the existence of the corresponding Berezin-Toeplitz star product was established. In 80 all star products "with separation of variables" on an arbitrary Kähler manifold were classified and in [81] the Berezin-Toeplitz star product was completely identified in terms of this classification. In [66] M. Engliš showed the existence of Berezin star-product on a quite general class of inhomogeneous complex domains. BerezinToeplitz quantization was recently studied by microlocal methods developed in 89. and 65. Applications of Berezin-Toeplitz quantization in the topological quantum field theory were given in [1].

Much work has been done to generalize Berezin's quantization on Kähler manifolds to other spaces. Berezin's first doctoral student Vladimir Molchanov developed harmonic analysis and quantization on para-Hermitian symmetric spaces (see 94, 95). Berezin's quantization on quantum Cartan domains was considered in [16]. Berezin's quantization was generalized to supermanifolds in [61, 73]. In the framework of this publication it is impossible to give a comprehensive survey of the growing body of papers building upon Berezin's work on quantization and many important papers are inevitably left out.

\section{Supermathematics}

5.1. Introductory remarks. Without doubt, Berezin is the creator of supermathematics, though it was not him who introduced the name. (More about the origin of the name in 5.4.) In hindsight, it is possible to trace the origins of what became supermathematics in various areas of pure mathematics and theoretical physics, but it only due to Berezin's vision and his conscious effort that these previously disjoint pieces became parts of a great unified picture together with a lot of new mathematics discovered by Berezin himself and by those who followed him. Speaking about Berezin's work in supermathematics, it is worth pointing out that it was interrupted by his untimely death when supermathematics was still in the early stages of its development; therefore, the loss caused by Berezin's sudden departure was greater for supermathematics than for other areas of his work.

Berezin's publications related to supermathematics can be divided into two groups corresponding to the two periods: the gestation period (1961-1975) and the 'super' period 19 (1975-1980).

We can formulate the main idea of supermathematics as follows. The systematic consideration of $\mathbb{Z}_{2}$-graded objects such as Abelian groups, vector spaces, algebras and modules with the corresponding sign convention ("Koszul's sign rule"20) allows

\footnotetext{
${ }^{18}$ Berezin-Toeplitz quantization is defined in terms of operators with given contravariant symbols. Such operators are generalizations of Toeplitz operators.

${ }^{19}$ The 'super' period is marked by the emergence of the names such as supermanifold, superalgebra, etc. As the borderline we may take the discovery of supersymmetric physical models followed by the introduction of the notion of a supermanifold in mathematics. This division is partly conventional.

${ }^{20}$ From an abstract viewpoint, the sign rule used in supermathematics is a very special example of a "commutativity constraint" or "braiding" in tensor categories.
} 
to construct a natural extension of the 'usual' linear algebra including generalizations of commutative algebras and Lie algebras. This goes further to the extension of differential and integral calculus of many variables and, geometrically, to the extensions of the notions of differentiable manifold, Lie group, algebraic variety (or scheme) and algebraic group.

Two things should be said.

Firstly, Berezin came to his program of supermathematics (without such a name, which appeared later) motivated by physics, more precisely, by his studies of the formalism of second quantization, which lies in the foundations of quantum field theory. The influence of physics was also decisive for the passage of supermathematics from its gestation stage to the modern stage.

Secondly, the 'supermathematical' generalization of the usual notions is not arbitrary, but indeed reflects the nature of things: the 'superanalogs' of various objects fit together in the same way as their prototypes do (but may also show non-trivial new phenomena). Moreover, this generalization is rigid and unique. There are no known further generalizations based on other gradings or more complicated commutativity constraints. That is, there are quantum groups and quantum spaces; however, they are isolated examples unified philosophically but not by a general theory such as a (non-existing) 'quantum' or 'braided' geometry, although these terms are sometimes applied.

5.2. Analysis on a Grassmann algebra. As already said, Berezin's program of supermathematics has its roots in his book [14] and the related articles [11, 48, 12, 13. (See the historical remarks in 3.5.) In order to construct a 'calculus of functionals' for the Fermi fields that can be parallel to the functional calculus used for describing the Bose fields, Berezin introduced differentiation and integration on a Grassmann algebra. He did it first for the Grassmann algebras with finite numbers of generators and then extended the results to the infinite-dimensional 'functional' case that he needed for his problem. This calculus allowed Berezin to obtain a 'functional realization' of the fermionic Fock space similar to the realization of the bosonic Fock space by holomorphic or antiholomorphic functions (or functionals) and to construct the spinor representation of the canonical transformations in the fermionic case (i.e., the spinor representations of certain infinite-dimensional versions of the orthogonal group). These representations were discussed in Section 3 and we shall not repeat it here.

A striking feature of Berezin's calculus on a Grassmann algebra was, as he noted in 14, that "differently from the usual rule for a change of variables, the independent variables and their differentials transform by reciprocal matrices". Here Berezin refers to his formulas

$$
\int x d x=1, \quad \int d x=0
$$

(where $x$ is a Grassmann generator), which imply $d(a x)=\frac{1}{a} d x$. The 'differential $d x^{\prime}$ is the quantity appearing under the integral sign, which we would now call the Berezin volume element and denote by $D x$ to distinguish it from the genuine differential of the variable $x$. We can see here the origins of superdeterminant (now called Berezinian), which was discovered some years later 21. Another remarkable

\footnotetext{
${ }^{21}$ When only odd variables are present, the Berezinian of their linear homogeneous transformation reduces simply to the inverse of the determinant.
} 
fact noted and used by Berezin in [14] was the appearance of the (square root of the) determinant of the quadratic form for a 'fermionic Gaussian integral' in the numerator, not in the denominator:

$$
\int e^{\sum a_{i k} x_{i} x_{k}} d x_{n} \ldots d x_{1}=\left(\operatorname{det}\left\|2 a_{i k}\right\|\right)^{1 / 2},
$$

where $a_{i k}=-a_{k i}$, in a sharp contrast with the familiar ("bosonic") case (equation 3.16 in [14]).

Integration on a Grassmann algebra introduced by Berezin was soon applied by Faddeev and Popov in their famous work on quantization of the Yang-Mills field: they expressed the Jacobian factor arising from the separation of the gauge degrees of freedom by a fermionic Gaussian integral over 'ghosts' (the 'Faddeev-Popov ghosts") and thus they were able to deduce the Feynman rules including ghosts as following from a local Lagrangian field theory. By contrast, Bryce DeWitt, who obtained close results at the same time, did not know Berezin's integration and because of that failed to arrive to such a natural formulation 22 .

At this point it makes sense to discuss the question about Berezin's predecessors. It is sometimes claimed that the use of anticommuting variables for the classical description of fermions was familiar to quantum physicists since 1950s (and hence Berezin did not introduce anything particularly new). Typically Schwinger's name is mentioned in this regard. In reality, the ideas of Schwinger and his disciples such as DeWitt about anticommuting variables were quite vague and did not go any further than the introduction of 'left' and 'right' derivatives with respect to generators of a Grassmann algebra (see, e.g., [108, 109]). Partial differentiation with respect to exterior generators had been already known to Élie Cartan in connection with his method of repère mobile (physicists were probably unaware of that). The novelty of Berezin's work in comparison with earlier and simultaneous works by physicists was in the mathematical clarity and power with which he developed the analogy between usual functions and elements of a Grassmann algebra, but the main new feature was integration over Grassmann generators with its striking properties. There is a saying 23 that "derivatives are algebra; analysis begins with integrals". It was Berezin who made this decisive step. It took some time for this achievement to be absorbed by the physical community: the example of DeWitt is a clear evidence.

One person who can be counted as a true predecessor of Berezin, is the British physicist J. L. Martin. In two papers 90, 91 of 1959, Martin introduced the notion of a general Hamiltonian system on a Poisson manifold (in the modern terminology) and suggested to extend it to more general algebras; in particular, he showed how to introduce what we would call a Poisson superalgebra structure on a Grassmann algebra and applied that to obtain a Lagrangian classical counterpart of a quantum particle of spin $1 / 2$; in the second paper, he started from a general algebraic formalism linking matrix calculus with nilpotent variables and applied it to constructing a Feynman integral over histories for fermionic systems. With hindsight, we may observe that Martin in these two works published together introduced the integral over anticommuting variables. Strangely, he applied the name 'integral' only for the functional case treated in 91]. For the finitedimensional case, he spoke about cosets modulo total differentials in [90] or an unnamed

\footnotetext{
${ }^{22}$ DeWitt explicitly admits that in the preface to the Russian translation (1985) of his influential book 'The dynamic theory of groups and fields'.

${ }^{23}$ I learned it from A. A. Kirillov.
} 
'operation $S_{\lambda}$ ' in [91, $\lambda$ being a Grassmann algebra generator or, more generally, an abstract nilpotent variable. Martin did not consider transformations of variables and the corresponding properties of the integral. It is amazing that the remarkable works [90, 91] were not continued and remained completely unnoticed. (Berezin learned about them only around 1976, see 46. He gives a very generous reference to 90, in the first sentence of 35.)

5.3. From Grassmann algebras to supermanifolds. Berezin's calculus on a Grassmann algebra as constructed in 11, 48, 12, 13] was not yet supermathematics in the proper sense. Ordinary variables and Grassmann algebra generators were considered in parallel but still separately. There was no mixture of them nor transformations of Grassmann variables other than linear. However, as Berezin described it later, "the striking coincidence of the main formulas of the operator calculus in the Fermi and Bose variants of the second quantization method... led to the idea of the possibility of a generalization of all the main notions of analysis so that generators of a Grassmann algebra would be on an equal footing with real or complex variables" 35, 38.

This was the program of supermathematics 24 .

The main steps of its implementation were as follows.

In [17, Berezin considered non-linear transformations of anticommuting variables in a clear departure from the standard viewpoint on the exterior algebra as a $\mathbb{Z}$-graded object associated with a linear space. Now the emphasis is shifted to the algebra itself and the transformations are supposed to preserve only $\mathbb{Z}_{2}$-grading and not necessarily $\mathbb{Z}$-grading. Berezin studied the effect of such transformations on the integral over anticommuting variables and proved that there appears the inverse of the determinant of the Jacobi matrix. This was a generalization of the formula in 14 and a step towards the discovery of Berezinian. No mixture with "ordinary" variables yet, but the whole logic leads in this direction.

Algebras generated by even and odd variables appeared in a joint paper of Berezin and G. I. Kac 43, who introduced - in modern language - formal Lie supergroups and Lie superalgebras and established their 1-1 correspondence. They used the results of Milnor and Moore 92. It should be said that a version of Lie superalgebras where the $\mathbb{Z}_{2}$-grading arises as the reduction of a $\mathbb{Z}$-grading modulo 2 had been long familiar to topologists and differential geometers under the confusing name of "graded Lie algebras" 25. The understanding that graded (co)commutative Hopf algebras played the role of the corresponding group objects was topologists' folklore 26. In algebraic topology, Hopf algebras arise as homology or cohomology of topological spaces, so in that context $\mathbb{Z}$-grading is natural. Unlike that, the algebras considered in 43 . were supposed to play the role of algebras of functions and the natural grading is $\mathbb{Z}_{2}$. Though [43] was devoted to the analogs of formal groups, the authors explained what the analog of a non-formal Lie group should be and gave two examples: in modern language, the general linear supergroup $\mathrm{GL}(n \mid m)$ and the diffeomorphism supergroup $\operatorname{Diff}\left(\mathbb{R}^{0 \mid m}\right)$.

\footnotetext{
${ }^{24}$ Of course, the name came after the program was actually fulfilled.

${ }^{25} \mathrm{~A}$ possible confusion with the ordinary Lie algebras possessing a grading.

${ }^{26}$ As S. P. Novikov told to the writer of these words many years ago, much of Milnor and Moore's paper had been part of folklore before its publication.
} 
(The notion of a Hopf algebra was discovered by Milnor, motivated by the study of cohomology operations. G. I. Kac, who should not be confused with V. G. Kac of KacMoody algebras, independently came to a close notion, which he called a 'ring group', working in representation theory. It was instrumental for his generalization of the Pontrjagin duality and the Tannaka-Krein duality. Works of G. I. Kac, who died untimely in 1978, anticipated the discovery of quantum groups; incidentally, before quantum groups no good examples of Hopf algebras that are neither commutative nor cocommutative were known. So the collaboration of Berezin and G. I. Kac on 43] was not accidental. Before [43, Nijenhuis came very close to the concept of a Lie supergroup in deformation theory. Nijenhuis used pairs consisting of a Lie superalgebra - of course, $\mathbb{Z}_{2}$ was $\mathbb{Z}$ modulo 2 - and a Lie group corresponding to its even part, which is an ordinary Lie algebra. Such pairs are equivalent to Lie supergroups and are nowadays sometimes referred to by the name 'Harish-Chandra pairs', borrowed from representation theory.)

After [17, 43, everything seems ready for the introduction of "spaces" for which the elements of $\mathbb{Z}_{2}$-graded algebras would be "functions". But in fact it required a few more years and some extra steps.

Such "spaces" remain implicit in paper [79] by G. I. Kac and A. I. Koronkevich, submitted shortly after [43, where a superanalog of Frobenius theorem in the language of differential forms was stated and proved.

A preliminary step was made in the setting of algebraic geometry. In paper [87, submitted in February 1973, Berezin's student D. A. Leites introduced a generalization of affine schemes (over a field) to the case of $\mathbb{Z}_{2}$-graded algebras. In particular, he introduced affine group schemes in this context and defined their Lie algebras (in the sense of 43 , i.e, Lie superalgebras).

The missing ingredient-before differentiable supermanifolds would become possible - was transformations of variables mixing the ordinary variables with Grassmann generators. Berezin came to the idea of such transformations studying his integral: about the same time as 43 was written, he arrived at a formula for a general change of variables in the integral over a collection of anticommuting and ordinary variables. According to Minlos 93], the conjectural statement originally appeared in 1971 in a letter to G. I. Kac. It contained, in particular, the notion of a 'superdeterminant' (this name emerged only later 27):

$$
\operatorname{sdet}\left(\begin{array}{ll}
A & B \\
C & D
\end{array}\right):=\operatorname{det}\left(A-B D^{-1} C\right)(\operatorname{det} D)^{-1} \text {. }
$$

Here the entries of $A, D$ are even and the entries of $B, C$, odd. The change of variables formula reads (in the notation close to Berezin's own notation):

$$
\int f(y, \eta) d \eta d y=\int f(y(x, \xi), \eta(x, \xi)) J(x, \xi) d \xi d x
$$

where

$$
J(x, \xi)=\operatorname{sdet}\left(\begin{array}{ll}
\frac{\partial y}{\partial x} & \frac{\partial \eta}{\partial x} \\
\frac{\partial y}{\partial \xi} & \frac{\partial \eta}{\partial \xi}
\end{array}\right) .
$$

The integral is extended to all values of the variables $y$ and the function $f(y, \eta)$ must be vanishing at the infinity of $y$.

It is a curious fact that Berezin did not publish the definition of superdeterminant and the change of variables formula himself. Berezin suggested a proof of (13) as

\footnotetext{
${ }^{27}$ Now the term 'Berezinian' and the notation Ber are universally adopted.
} 
a problem to his student V. F. Pakhomov, in whose paper [103, submitted in December 1973, the above formulas first appeared in print 28 .

Allowing changes of variables in the Berezin integral implied considering ordinary coordinates and Grassmann generators on an equal footing as generators of the algebra $C^{\infty}\left(\mathbb{R}^{n}\right) \otimes \Lambda\left(\mathbb{R}^{m}\right)$, denoted $\mathfrak{B}_{n, m}$ in [103] (in modern language it is $\left.C^{\infty}\left(\mathbb{R}^{n \mid m}\right)\right)$. This was probably the final step towards supermanifolds.

An algebraic proof of the multiplicativity of "Berezin's function" (12) was given by D. A. Leites in a short note 88, submitted in May 1974.

\subsection{Emergence of supersymmetric models and the explicit introduction}

of supermanifolds. The analysis in the previous subsection amply demonstrates that Berezin's program had been mainly fulfilled by himself and his collaborators by around 1973. The notion of a supermanifold was for them "in the air", though it had not appeared in the publications explicitly. The same can be said about supergroups. Still, according to Leites, Berezin felt reluctant to publish the definition of a supermanifold and was forced to do so only in order not to lose the priority. So what happened?

The momentum came again from theoretical physics and it was supersymmetry. Now this name is used very widely and sometimes outside of its precise original meaning, which is transformations of fields mixing the fermionic fields (usually describing 'matter') with bosonic fields (usually describing 'interaction').

In parallel with Berezin's work, the breakthrough was preparing in 1971-1974. Supersymmetry appeared, in the context of 'dual-resonance models' (later, string theory), in Ramond [104] and Neveu-Schwarz [100; and, in the context of fourdimensional gauge theory, in Golfand-Likhtman [72, Volkov et al. [119, 120, 121] (who explicitly quoted Berezin and G. I. Kac 43]), and finally in Wess-Zumino [123, 124, whose work resulted in an explosion. Physicists started to look around for mathematical foundations of the new theory. Salam and Strathdee 105 were the first to formulate the concept of a 'superspace' on an operational level.

In such a context, Berezin was forced to act quickly. Berezin and Leites published [45]. This paper contained the definition of a supermanifold as a local ringed space modeled on open domains of $\mathbb{R}^{p}$ endowed with the $\mathbb{Z}_{2}$-graded algebra $C^{\infty}\left(\mathbb{R}^{p}\right) \otimes \Lambda\left(\mathbb{R}^{q}\right)$; the notions of morphisms of supermanifolds, subsupermanifolds and the direct products; the coordinate description by local charts and coordinate transformations; the notion of what we now call Berezin volume density and the construction of the Berezin integral over a supermanifold; specifying supermanifolds by equations in $\mathbb{R}^{n \mid m}$ and a conjecture that this may be possible for an arbitrary supermanifold (analog of Whitney's theorem); Lie supergroups (global) and their Lie superalgebras (so renamed from the 'Lie algebras' of [43]). Quite a lot! Of course, the big work remained to elaborate the details and to make them available to the public.

It is time to say something about the terminology which involves the prefix 'super-': supermanifold, superalgebra, supergroup, superspace... In physical context, the term "supersymmetry" has a direct meaning as a "superior symmetry" exceeding other symmetries that keep bosons and fermions separate. In mathematics, the prefix 'super-' should be understood as an abbreviation from supersymmetry,as having something to do with physical supersymmetries. Berezin himself did not

${ }^{28}$ Without any particular name and notation for the function sdet. 
overuse this prefix. It was done by others, and this made an unfortunate aftertaste. Nevertheless, such is the universally adopted terminology and there is no other choice but follow it.

5.5. Berezin's work on supermathematics in 1975-1980. There were several directions of Berezin's work after the introduction of supermanifolds.

The physical papers by Berezin and Marinov [46, 47] were devoted to the description of spin by means of supermathematics. These works are still on the borderline with the previous period: they do not use the word 'supermanifold' yet; the earlier paper [46] was submitted for publication just one month after [45]. There is an interesting historical material (mainly physical) in [47, in particular, references to Martin 190, 91. Berezin came to this subject again in a joint paper with V. L. Golo 41] of 1980. It appeared only a few days before Berezin died; he could not see it published. One can also mention here the posthumous publication [42] on a chiral supersymmetric sigma-model.

A central topic of Berezin's research in 1975-1980 was the theory of Lie superalgebras and Lie supergroups, especially their representations and invariants. Berezin's methods were global, geometric and analytic (e.g., used tools such as invariant integral) rather than infinitesimal.

In a short article 27, Berezin studied the Lie supergroup $\mathrm{U}(p \mid q)$ and its unitary representations 29. In particular, Berezin found the invariant integral and the radial parts of Laplace operators; he introduced "non-degenerate" or "typical" irreducible representations and found their characters.

The method sketched in 27 was elaborated and generalized in a series of five preprints [28, 29, 30, 31, 32 of 1977. They contain very interesting material; it would be fair to say that much of it has unfortunately remained not well understood yet. These preprints, originally published in a small number of copies, were later included in the expanded English edition 30 of 38 .

Two joint papers of Berezin and V. S. Retakh [51, 52] of 1978 were devoted to classification of Lie superalgebras whose even part is semisimple. (The classification of simple Lie superalgebras over $\mathbb{C}$ was obtained by V. G. Kac around 1975 , who interacted actively with Berezin at that time. Kac's classification remarkably brings forward superanalogs of classical matrix Lie algebras and the Lie algebras of vector fields, which is one more evidence for the "naturalness" of supergeometry.)

Berezin's last publication on representations of Lie supergroups was the paper with V. N. Tolstoy [56] dealing with a certain real compact form of the Lie supergroup $\operatorname{OSp}(1 \mid 2)$. (It appeared already after his death.)

Besides the Lie supergroups, Berezin actively worked on the general theory of supermanifolds. We should mention the expository preprint [33] and the survey paper [35] (both of 1979), and of course Berezin's work on a book on supermanifolds, which was incomplete at the time of his death. It was to appear only as a posthumous publication [38, compiled and edited by his friends such as A. A. Kirillov and V. P. Palamodov. (Palamodov, in particular, included there his own new results on the structure of supermanifolds.) The Russian version appeared in 1983 and the expanded English translation in 1987.

\footnotetext{
${ }^{29}$ Berezin's own notation for this Lie supergroup was $U(p, q)$ and this should not lead to a confusion with the ordinary Lie group of pseudounitary matrices.

${ }^{30} \mathrm{We}$ should be thankful to the editors for that. The quality of the English translation is sometimes poor: e.g., "resultant" is confused with "result", but it is still readable.
} 
Three mathematical questions that attracted Berezin's attention are worth mentioning.

The first was the question about "points of supermanifolds". No doubt, the fact that "functions" on supermanifolds contain nilpotents makes it harder to understand them as compared to ordinary manifolds. Supermanifolds cannot be treated as sets with some structure. For example, the supermanifold $\mathbb{R}^{0 \mid m}$, whose "algebra of functions" is the Grassmann algebra with $m$ generators, set-theoretically consists of a single point; clearly, the structure of $\mathbb{R}^{0 \mid m}$ cannot be attributed to this one-point set. At the same time, physicists working with "superspace" freely used "points" such as $\left(x^{a}, \xi^{\mu}\right)$ with the odd coordinates $\xi^{\mu}$, whatever that could mean. Berezin's solution to that was in the introduction of an auxiliary Grassmann algebra $\mathfrak{G}(N)$ with a large or infinite number of generators $N$ and in considering, for each supermanifold $M$, the ordinary manifold $M(N)$ (of large dimension) obtained by replacing abstract even and odd coordinates in the coordinate transformations for $M$ by elements of $\mathfrak{G}(N)$ of corresponding parities. The manifold $M(N)$, by construction, has a special structure called by Berezin a Grassmann-analytic structure 31 . If $N$ is large enough, the supermanifold $M$ can be recovered from $M(N)$ taken with this structure. At the same time, the 'Grassmann-analytic manifold' $M(N)$ is a set-theoretic object and can be described by its points. Berezin used this idea widely, in particular for representations of Lie superalgebras and Lie supergroups, which he replaced by (in his terminology) their 'Grassmann envelopes'.

Berezin's idea about the manifolds $M(N)$ and 'Grassmann-analytic manifolds' in general contained the roots of several later developments (some probably independent of him). If one fixes a Grassmann algebra $\mathfrak{G}(N)$ and considers manifolds over it endowed with some class of 'Grassmann-analytic functions', there is a temptation to forget about supermanifolds defined as ringed spaces altogether. Two versions of this idea were put forward, by B. DeWitt and by Alice Rogers, but in spite of all intuitive attractiveness, it was later found that its consistent development takes one back to the sheaf-theoretic approach to supermanifolds. Another option is not to fix $\mathfrak{G}(N)$, but consider the manifolds $M(N)$ as a functor of $\mathfrak{G}(N)$. Together they represent the original supermanifold $M$. This was suggested by A. S. Schwarz. It allows to consider objects more general than supermanifolds.

The second question concerned general classification of supermanifolds. It is obvious that the case in which the coordinate changes for a given supermanifold do not mix odd variables with the even variables and the odd variables transform linearly is the simplest and it corresponds to a vector bundle over an ordinary manifold. The question is how general this case is, i.e., whether it is always possible to reduce coordinate transformations to this simple form by a choice of atlas. The answer is, yes, - for smooth supermanifolds. This statement is often referred to as the Batchelor theorem after Marjorie Batchelor who proved it in 1979. No doubt that Berezin knew it independently: he mentions it in [33, 35]. As for complex-analytic supermanifolds, the answer is, no; there are obstructions. The corresponding theory and examples of "non-retractable" complex-analytic supermanifolds are due to V. P. Palamodov (a slightly different approach was developed by Yu. I. Manin).

\footnotetext{
${ }^{31}$ This exactly means that the coordinate transformations on $M(N)$ are given by transformations of elements of the algebra $\mathfrak{G}(N)$ taken as whole quantities-like complex numbers instead of their real and imaginary parts taken separately. This is a particular case of what is called a 'manifold over an algebra'.
} 
Finally, the third question concerned integration on supermanifolds and differential forms. It is clear that Berezin's transformation law for the element of volume is different from what one gets for the differentials of coordinates defined in a straightforward way. So on supermanifolds, differential forms and integration theory seem to split. The problem was tackled by J. N. Bernstein and D. A. Leites, who introduced 'integral forms' [57, incorporating volume elements, as a replacement of differential forms for the purpose of integration and 'pseudodifferential forms' 58 as not necessarily polynomial expressions in differentials (which also opens way for integration).

Berezin in [36] introduced a further generalization of the Bernstein-Leites pseudodifferential forms, studied their duality transformations and sketched a Weil-type construction of characteristic classes. Berezin's aim was future application to super gauge theory. Paper [36] seems to be Berezin's last paper on supermanifolds. It is worth mentioning that [36] contained a construction very close to what is now known as the 'homological interpretation' of Berezinian.

5.6. Influence. Later developments. The influence of Berezin's work on supermathematics remains different in physics and in pure mathematics. Physicists have completely absorbed the idea of working with supermanifolds. For them, supergeometry is a tool on the same footing as tensor calculus: physicists use it without even noticing it. Unlike that, in pure mathematics, Berezin's ideas have spread far less widely. Supermanifolds for many remain something exotic (except for those directly working in supergeometry). Quite characteristic is that representations of Lie superalgebras became a well-established area, but those working in it rarely consider Lie supergroups or turn to global methods used by Berezin. No doubt, the landscape would be quite different, had Berezin not died in 1980. However, the situation is slowly changing. "Supermethods" start to spread in differential geometry. Of course, this development is more significant in areas closer to or more influenced by physics. Two Fields medals awarded in 1990 and 1998, to E. Witten and M. L. Kontsevich, respectively, were related with works where supergeometry played a role. (Morse theory and differential forms, in the case of Witten, and deformation theory and quantization of Poisson manifolds, in the case of Kontsevich.)

One general trend worth mentioning is a certain shift from "supersymmetry" (roughly, transformations squaring to ordinary symmetries) to "BRST-symmetry" and " $Q$-manifolds" (where, roughly, there are transformations with square zero). A central role has been played here by the Batalin-Vilkovisky formalism in quantum field theory [3, 4, 5] and its modern generalizations. Geometrically, that means considering supermanifolds endowed with an odd symplectic structure and odd Laplacians on them. (The study of such geometry was pioneered by H. M. Khudaverdian 82, see also 83, 84.)

Another trend is the growth of importance of graded manifolds - not in the sense synonymous with supermanifolds as the usage in the early period sometimes was 32 , - but meaning supermanifolds endowed with extra $\mathbb{Z}$ - or $\mathbb{Z}_{+}$-grading, which in physics may be for example, 'ghost number'. If one recalls topologists' $\mathbb{Z}$-graded algebras and the replacement of $\mathbb{Z}$-grading by $\mathbb{Z}_{2}$-grading as a step in development

\footnotetext{
${ }^{32}$ In the Western literature, when the foundations of supermanifolds were thought to be not fully established yet, 'supermanifolds' were often used for DeWitt or Rogers's versions of manifolds over Grassmann algebras, while 'supermanifolds' in Berezin's sense were called 'graded manifolds'.
} 
of supermathematics, as described above, the reintroduction of $\mathbb{Z}$-gradings (but now as additional structure) completes the circle, but at a higher level.

We would like to finish this section by two interesting pieces of mathematics related with Berezin integration and Berezinian (superdeterminant), which were discovered after Berezin.

In the previous subsection, we considered the works on integration theory and differential forms by Bernstein-Leites and by Berezin [36. In search of objects suitable for integration over (multidimensional) paths or surfaces in supermanifolds, a variational approach to "forms on supermanifolds" was developed (Th. Th. Voronov and A. V. Zorich, see [122]; building on earlier works by A. S. Schwarz and his students): analogs of forms were constructed as Lagrangians satisfying certain restrictions. An amazing fact discovered along these lines and not fully understood yet is the following link with integral geometry in the sense of Gelfand-Gindikin-Graev: the equation of the form 33

$$
\frac{\partial^{2} f}{\partial w_{a}^{i} \partial w_{b}^{j}}+(-1)^{\tilde{\imath} \tilde{\jmath}+\tilde{a}(\tilde{\imath}+\tilde{\jmath})} \frac{\partial^{2} f}{\partial w_{a}^{j} \partial w_{b}^{i}}=0,
$$

for a function of a rectangular supermatrix $\left\|w_{a}^{i}\right\|$, arises in the de Rham theory on supermanifolds as a condition replacing skew-symmetry and multilinearity (see [122]) and at the same time it is a generalization of 'hypergeometric equations' in the sense of Gelfand (the odd-odd part of (15) is the F. John equation arising in relation with the Radon transform).

Another beautiful development related with the notion of Berezinian is as follows. Th. Schmitt 107 discovered that the expansion of Berezinian leads to exterior powers:

$$
\operatorname{Ber}(1+z A)=1+z \operatorname{str} A+z^{2} \operatorname{str} \Lambda^{2} A+\ldots .
$$

Here $A$ is an even supermatrix,

$$
A=\left(\begin{array}{ll}
A_{00} & A_{01} \\
A_{10} & A_{11}
\end{array}\right)
$$

$\Lambda^{k} A$ stands for its action on the $k$ th exterior power and str denotes the supertrace: $\operatorname{str} A=\operatorname{tr} A_{00}-\operatorname{tr} A_{11}$. As it was found in 85, by comparing the expansions of $\operatorname{Ber}(1+z A)$ at zero and at infinity one arrives at certain universal recurrence relations satisfied by the differences of the respective coefficients. In particular, for a $p|q \times p| q$ matrix, there are relations

$$
\left|\begin{array}{ccc}
c_{k}(A) & \ldots & c_{k+q}(A) \\
\ldots & \ldots & \ldots \\
c_{k+q}(A) & \ldots & c_{k+2 q}(A)
\end{array}\right|=0,
$$

where $c_{k}(A)=\operatorname{str} \Lambda^{k} A$, satisfied for all $k>p-q$. (This replaces the vanishing of the $k$ th exterior powers for an $n$-dimensional space with $k>n$ ). Similar relations hold in the Grothendieck ring of a general linear supergroup, and there is a formula

$$
\operatorname{Ber} A=\frac{\left|c_{p-q}(A) \ldots c_{p}(A)\right|_{q+1}}{\left|c_{p-q+2}(A) \ldots c_{p+1}(A)\right|_{q}}
$$

\footnotetext{
33 The tilde over an index denotes the parity of the corresponding variable.
} 
with Hankel's determinants at the top and at the bottom, expressing Berezinian as the ratio of polynomial invariants 34 .

Acknowledgment. We thank S. G. Gindikin for discussions. A. V. Karabegov and Yu. A. Neretin acknowledge the support by the NSF and RFBR respectively that made their participation in the XXXth Białowieża conference possible. (The NSF award number: 1124929.) Neretin's work was also partially supported by the FWF grant, Project 22122.

\section{REFERENCES}

[1] J. E. Andersen and J. Blaavand. Asymptotics of Toeplitz operators and applications in TQFT. Travaux Mathematiques 19 (2011), 167-201.

[2] V. Bargmann. On a Hilbert space of analytic functions and an associated integral transform. Comm. Pure Appl. Math. 14 (1961), 187-214.

[3] I. A. Batalin and G. A. Vilkovisky. Gauge algebra and quantization. Phys. Lett. 102B (1981), 27-31.

[4] I. A. Batalin and G. A. Vilkovisky. Quantization of gauge theories with linearly dependent generators. Phys. Rev. D28 (1983), 2567-2582.

[5] I. A. Batalin and G. A. Vilkovisky. Closure of the gauge algebra, generalized Lie equations and Feynman rules. Nucl. Phys. B 234 (1984), 106-124.

[6] F. Bayen, M. Flato, C. Fronsdal, A. Lichnerowicz, and D. Sternheimer. Deformation theory and quantization. I. Deformations of symplectic structures. Ann. Physics 111 (1978), no. 1, $61-110$.

[7] F. A. Berezin. Laplace operators on semisimple Lie groups. Dokl. Akad. Nauk SSSR (N.S.) 107 (1956), 9-12.

[8] F. A. Berezin. Representation of complex semisimple Lie groups in Banach space. Dokl. Akad. Nauk SSSR (N.S.) 110 (1956), 897-900.

[9] F. A. Berezin. Laplace operators on semisimple Lie groups and on certain symmetric spaces. (Russian) Uspehi Mat. Nauk (N.S.) 12 (1957), no. 1(73), 152-156. (Translated in: Amer. Math. Soc. Transl. (2) 16 (1960), 364-369.)

[10] F. A. Berezin. Laplace operators on semisimple Lie groups. Trudy Moskov. Math. Obshchestva. 6 (1957), 371-463. (Translated in: Amer. Math. Soc. Transl. (2) 21 (1962), 239-339.)

[11] F. A. Berezin. Canonical operator transformation in representation of secondary quantization. Dokl. Akad. Nauk SSSR 137, 311-314 (Russian); translated as Soviet Physics Dokl. 6 (1961), 212-215.

[12] F. A. Berezin. Canonical transformations in the second quantization representation. (Russian) Dokl. Akad. Nauk SSSR 150 (1963), 959-962.

[13] F. A. Berezin. Operators in the representation of secondary quantization. Dokl. Akad. Nauk SSSR 154, 1063-1065 (Russian); translated as Soviet Physics Dokl. 9 (1964), 142-144.

[14] F. A. Berezin. The Method of Second Quantization. Nauka, Moscow, 1965. Tranlation: Academic Press, New York, 1966. (Second edition, expanded: M. K. Polivanov, ed., Nauka, Moscow, 1986.)

[15] F. A. Berezin. Some remarks on the representations of commutation relations. Uspehi Mat. Nauk 24, no. 4 (148) (1969), 65-88; Russian Math. Surv. 24, No.4, 65-88.

[16] F. A. Berezin. Letter to the editor. Trudy Moskov. Mat. Obshch. 12 (1963), 453-466.

[17] F. A. Berezin. Automorphisms of a Grassmann algebra. Mathematical Notes, 1 (1967), 180-184.

[18] F. A. Berezin. Some remarks about the associative envelope of a Lie algebra. Funct. Anal. Appl. 1 (1967), 91-102.

[19] F. A. Berezin. Non-Wiener path integrals. (Russian) Teoret. Mat. Fiz. 6 (1971), no. 2, 194-212.

[20] F. A. Berezin. Wick and anti-Wick operator symbols. Mat. Sb. (N.S.) 15 (1971), 577-606.

\footnotetext{
${ }^{34}$ In the definition, $\operatorname{Ber} A=\operatorname{det}\left(A_{00}-A_{01} A_{11}^{-1} A_{10}\right) \operatorname{det} A_{11}^{-1}$, neither the numerator nor the denominator of the fraction are invariant.
} 
[21] F. A. Berezin. Covariant and contravariant symbols of operators. Math. USSR-Izv. 36 (1972), 1134-1167.

[22] F. A. Berezin. Convex operator functions. Mat. Sb. (N.S.) 17 (1972), 269-278.

[23] F. A. Berezin. Quantization in complex bounded domains. (Russian) Dokl. Akad. Nauk SSSR 211 (1973), 1263-1266.

[24] F. A. Berezin. Quantization. Math. USSR-Izv. 38 (1974), 1116-1175.

[25] F. A. Berezin. General concept of quantization. Comm. Math. Phys. 40 (1975), 153-174.

[26] F. A. Berezin. Quantization in complex symmetric spaces. Math. USSR-Izv. 39 (1975), 363-402.

[27] F. A. Berezin. Representations of the supergroup $U(p, q)$. Funct. Anal. Appl 10(3) (1976), 221-223. (Transl. from: Funkcion. Anal. i Priložen., 10(3) (1976), 70-71.)

[28] F. A. Berezin. Lie superalgebras. Preprint ITEP-66, 1977.

[29] F. A. Berezin. Lie supergroups. Preprint ITEP-78, 1977.

[30] F. A. Berezin. Laplace-Casimir operators (general theory). Preprint ITEP-77, 1977.

[31] F. A. Berezin. The radial parts of the Laplace-Casimir operators on Lie supergroups $U(p, q)$ and $C(m, n)$. Preprint ITEP-75, 1977.

[32] F. A. Berezin. Construction of representations of Lie supergroups $U(p, q)$ and $C(m, n)$. Preprint ITEP-76, 1977.

[33] F. A. Berezin. Supermanifolds. Preprint ITEP, 1979.

[34] F. A. Berezin. The connection between covariant and contravariant symbols of operators on classical complex symmetric spaces. (Russian) Dokl. Akad. Nauk SSSR 241 (1978), no. 1, $15-17$.

[35] F. A. Berezin. The mathematical basis of supersymmetric field theories. Soviet J. Nuclear Phys., 29(6) (1979), 1670-1687.

[36] F. A. Berezin. Differential forms on supermanifolds. Soviet J. Nuclear Phys., 30(4) (1979), 605-609.

[37] F. A. Berezin. Feynman path integrals in a phase space. (Russian) Soviet Phys. Uspekhi 132 (1980), no. 3, 497-548.

[38] F. A. Berezin. Introduction to algebra and analysis with anticommuting variables. V. P. Palamodov, ed., Moscow State University Press, Moscow, 1983. Expanded transl. into English: Introduction to superanalysis. A. A. Kirillov, ed., D. Reidel, Dordrecht, 1987.

[39] F. A. Berezin and I. M. Gelfand. Some remarks on the theory of spherical functions on symmetric Riemannian manifolds. (Russian) Trudy Moskov. Mat. Obshch. 5 (1956), 311351.

[40] F. A. Berezin, I. M. Gelfand, M. I. Graev. Group representations. Uspehi Mat. Nauk (N.S.) 11 (1956), no. 6(72), 13-40. (Amer. Math. Soc. Transl. (2) 16 (1960), 325-353.)

[41] F. A. Berezin and V. L. Golo. Supersymmetric model of several classical particles with spin. (Russian) JETP Letters 32(1) (1980), 82-84.

[42] F. A. Berezin, V. L. Golo, and B. A. Putko. Reduction of a chiral supersymmetric $\sigma$-model. Vestnik Moskov. Univ. Ser. I Mat. Mekh., (3) (1982), 16-19, 109.

[43] F. A. Berezin and G. I. Kac. Lie groups with commuting and anticommuting parameters. Mathematics of the USSR-Sbornik, 11(3) (1970), 311-325.

[44] F. A. Berezin and F. I. Karpelevich. Zonal spherical functions and Laplace operators on some symmetric spaces. Dokl. Akad. Nauk SSSR 118 (1958), 9-12.

[45] F. A. Berezin and D. A. Leites. Supermanifolds. Soviet Math. Dokl. 16(5) (1975), 1218-1222.

[46] F. A. Berezin and M. S. Marinov. Classical spin and the Grassmann algebra. JETP Letters 21(11) (1975), 678-680.

[47] F. A. Berezin and M. S. Marinov. Particle spin dynamics as the Grassmann variant of classical mechanics. Annals of Physics 104 (1977), 336-362.

[48] F. A. Berezin, R. A. Minlos, and L. D. Faddeev. Some problems of quantum-mechanical systems with large number of the degrees of freedom. Proceedings of 4-th All-Union Congress of Mathematics (1961), Moscow, 1964, vol. 2, 532-541.

[49] F. A. Berezin and A. M. Perelomov. Group-theoretic interpretation of equations of the Korteweg-de Vries type. Funktsional. Anal. i Prilozhen. 14(2) (1980), 50-51.

[50] F. A. Berezin and A. M. Perelomov. Group-theoretical interpretation of the Korteweg-de Vries type equations. Comm. Math. Phys. 74(2) (1980), 129-140.

[51] F. A. Berezin and V. S. Retah. The structure of Lie superalgebras with a semisimple even part. Funkcional. Anal. i Priložen. 12(1) (1978), 64-65. 
[52] F. A. Berezin and V. S. Retah. Construction of Lie superalgebras with a semisimple even part. Vestnik Moskov. Univ. Ser. I Mat. Mekh. (5) (1978), 63-67.

[53] F. A. Berezin and V. S. Retakh. A method of computing characteristic classes of vector bundles. Rep. Math. Phys. 18(3) (1980), 363-378.

[54] F. A. Berezin and M. A. Shubin. Symbols of operators and quantization. In Hilbert space operators and operator algebras (Proc. Internat. Conf., Tihany, 1970), pages 21-52. Colloq. Math. Soc. János Bolyai, No. 5. North-Holland, Amsterdam, 1972.

[55] F. A. Berezin and M. A. Shubin. The Schrödinger equation. Moscow State University Press, Moscow, 1983. Transl. into English: Kluwer, Dordrecht, 1991.

[56] F. A. Berezin and V. N. Tolstoy. The group with Grassmann structure UOSP(1.2). Comm. Math. Phys. 78(3) (1980/81), 409-428.

[57] J. N. Bernstein and D. A. Leites. Integral forms and Stokes formula on supermanifolds. Funk. Anal. Pril. 11(1) (1977), 55-56.

[58] J. N. Bernstein and D. A. Leites. How to integrate differential forms on supermanifolds. Funk. Anal. Pril. 11(3) (1977), 70-71.

[59] N. N. Bogolyubov, I. M. Gelfand, R. L. Dobrushin, A. A. Kirillov, M. G. Krein, D. A. Leites, R. A. Minlos, Ya. G. Sinai, and M. A. Shubin. Feliks Aleksandrovich Berezin. Obituary. Uspekhi Mat. Nauk 36(4(220)) (1981), 185-190.

[60] M. Bordemann, E. Meinrenken, and M. Schlichenmaier. Toeplitz quantization of Kähler manifolds and $\operatorname{gl}(n), n \rightarrow \infty$, limits. Comm. Math. Phys. 165 (1995), 281-296.

[61] D. Borthwick, S. Klimek, A. Lesniewski, and M. Rinaldi. Super Toeplitz operators and non-perturbative quantization of supermanifolds, Commun. Math. Phys. 153 (1993), $49-$ 76.

[62] L. Boutet de Monvel and V. Guillemin. The spectral theory of Toeplitz operators. Ann. Math. Stud. 99, Princeton University Press, Princeton, 1981.

[63] M. Cahen, S. Gutt, and J. Rawnsley. Quantization of Kähler manifolds I: Geometric interpretation of Berezin's quantization. JGP 7 (1990), 45-62.

[64] M. Cahen, S. Gutt, and J. Rawnsley. Quantization of Kähler manifolds II. Trans. Amer. Math. Soc. 337 (1993), 73-98.

[65] L. Charles. Berezin-Toeplitz operators, a semi-classical approach. Comm. Math. Phys. 239 (2003), 1-28.

[66] M. Engliš. Weighted Bergman kernels and quantization. Comm. Math. Phys. 227 (2002), 211-241.

[67] J. Feldman. Equivalence and perpendicularity of Gaussian processes. Pac. J. Math. 8 (1958), 699-708.

[68] K. O. Friedrichs. Mathematical aspects of the quantum theory of fields. Interscience Publishers, Inc., New York, 1953.

[69] W. Fulton. Eigenvalues, invariant factors, highest weights, and Schubert calculus. Bull. Am. Math. Soc., New Ser. 37, No.3 (2000), 209-249.

[70] I. M. Gelfand and M. A. Naimark. Unitary representations of classical groups. Trudy Mat.Inst. Steklov., v.36 (1950) (Russian); German translation: I. M. Gelfand and M. A. Neumark. Unitare Darstellungen der klassischen Gruppen, Akademie-Verlag, Berlin, 1957.

[71] R. Godement. A theory of spherical functions. I. Trans. Am. Math. Soc. 73 (1952), 496-556.

[72] Yu. A. Golfand and E. P. Likhtman. Extension of the algebra of Poincaré group generators and violation of P invariance. JETP Letters 13 (1971), 323-326.

[73] A. El Gradechi and L. M. Nieto. Supercoherent States, Super Kähler Geometry and Geometric Quantization. Commun.Math.Phys. 175 (1996), 521-564.

[74] A. Guichardet and D. Wigner. Sur la cohomologie réelle des groupes de Lie simples réels. Ann. Sci. École Norm. Sup. (4) 11 (1978), no. 2, 277-292.

[75] Harish-Chandra. Representations of semisimple Lie groups, II. Trans. Amer. Math. Soc. 76 (1953), 26-65.

[76] Harish-Chandra. The characters of semisimple Lie groups. Trans. Amer. Math. Soc. 83 (1956), 98-163.

[77] G. Heckman and H. Schlichtkrull. Harmonic analysis and special functions on symmetric spaces. (English) Perspectives in Mathematics. 16. Orlando, FL: Academic Press, Inc. xii, $225 \mathrm{p}$.

[78] A. Huckleberry and M. Kalus. Selected results on Lie supergroups and their radial operators. Preprint: arXiv:1012.5233. 
[79] G. I. Kac and A. I. Koronkevič. The Frobenius theorem for functions of commuting and anticommuting arguments. Funkcional. Anal. i Priložen. 5(1) (1971), 78-80.

[80] A. Karabegov. Deformation quantizations with separation of variables on a Kähler manifold. Comm. Math. Phys. 180 (1996), 745-755.

[81] A. Karabegov and M. Schlichenmaier. Identification of Berezin-Toeplitz deformation quantization. J. reine angew. Math. 540 (2001), 49-76.

[82] H. M. Khudaverdian. Geometry of superspace with even and odd brackets. J. Math. Phys. 32 (1991), 1934-1937. (Preprint of the Geneva University, UGVA-DPT 1989/05-613, 1989.)

[83] H. M. Khudaverdian and Th. Th. Voronov. On odd Laplace operators. Lett. Math. Phys. 62 (2002), 127-142.

[84] H. M. Khudaverdian and Th. Th. Voronov. On odd Laplace operators. II. In book: Geometry, Topology and Mathematical Physics. S. P. Novikovs seminar: 2002 - 2003, V. M. Buchstaber and I. M. Krichever, editors, Amer. Math. Soc. Transl. (2), Vol. 212, 2004, pp.179-205.

[85] H. M. Khudaverdian and Th. Th. Voronov. Berezinians, exterior powers and recurrent sequences. Lett. Math. Phys. 74(2) (2005), 201-228.

[86] A. A. Klyachko. Stable bundles, representation theory and Hermitian operators. Selecta Math., New Ser. 4, No. 3 (1998), 419-445.

[87] D. A. Leites. Spectra of graded-commutative rings. Uspehi Mat. Nauk 29(3(177)) (1974), 209-210.

[88] D. A. Leites. A certain analogue of the determinant. Uspehi Mat. Nauk 30(3(183)) (1975), 156.

[89] X. Ma and G. Marinescu. Holomorphic Morse inequalities. Progress in Mathematics, 254 (2007), Birkhäuser Verlag, Basel.

[90] J. L. Martin. Generalized classical dynamics, and the "classical analogue" of a Fermi oscillator. Proc. Roy. Soc. London. Ser. A 251 (1959), 536-542.

[91] J. L. Martin. The Feynman principle for a Fermi system. Proc. Roy. Soc. London. Ser. A 251 (1959), 543-549.

[92] J. W. Milnor and J. C. Moore. On the structure of Hopf algebras. Ann. of Math. (2) 81 $1965,211-264$.

[93] R. A. Minlos. Felix Alexandrovich Berezin (a brief scientific biography). Lett. Math. Phys. 74(1) (2005), 5-19.

[94] V. F. Molchanov. Quantization on the imaginary Lobachevskii plane. Funkts. Anal. Prilozh., 14:2 (1980), 7374.

[95] V. F. Molchanov. Quantization on para-Hermitian symmetric spaces. Amer. Math. Soc. Transl., Ser. 2, 175 (1996), (Adv. in Math. Sci., 31), 81-95.

[96] C. Moreno. *-Products on some Kähler manifolds. Lett. Math. Phys. 11 (1986), 361-372.

[97] Yu. A. Neretin. Plancherel formula for Berezin deformation of $L^{2}$ on Riemannian symmetric space. J. Funct. Anal. 189, No. 2 (2002), 336-408.

[98] Yu. A. Neretin. "The method of second quatization": a view 40 years after.

In book: Recollections of Felix Alexandovich Berezin, the founder of supermathematics, Moscow, MCCME publishers, 2009 (Russian), available at http://www.mat.univie.ac.at/ neretin/zhelobenko/berezin.pdf Translation into French by C. Roger (with participation of O. Kravchenko, D. Millionschikov and A. Kosyak) is available at http://hal.archives-ouvertes.fr/docs/00/47/84/76/PDF/neretin.pdf

[99] Yu. A. Neretin. Lectures on Gaussian integral operators and classical groups. EMS Series of Lectures in Mathematics. Zürich: European Mathematical Society, 2011, xii, 559 p.

[100] A. Neveu and J. H. Schwarz. Factorizable dual model of pions. Nuclear Phys. B 31 (1971), 86-112.

[101] M. A. Olshanetsky and A. M. Perelomov. Completely integrable Hamiltonian systems connected with semisimple Lie algebras. Inv. Math. 37 (1976), 93-108.

[102] M. A. Olshanetsky and A. M. Perelomov. Quantum systems related to root systems, and radial parts of Laplace operators. Funct. Anal. Appl. 12 (1978), 121-128.

[103] V. F. Pahomov. Automorphisms of the tensor product of an abelian and a Grassmann algebra. Math. Notes 16 (1974), 624-629. Transl. from: Matem. Zametki 16 (1) (1974), 65-74.

[104] P. Ramond. Dual theory for free fermions. Phys. Rev. D (3) 3 (1971), 2415-2418. 
[105] A. Salam and J. Strathdee. Super-gauge transformations. Nuclear Phys. B 76 (1974), 477482.

[106] M. Schlichenmaier. Berezin-Toeplitz quantization of compact Kähler manifolds. In: Quantization, Coherent States and Poisson Structures, Proc. XIVth Workshop on Geometric Methods in Physics (Białowieża, Poland, 9-15 July 1995), A. Strasburger, S. T. Ali, J.-P. Antoine, J.-P. Gazeau, and A. Odzijewicz, eds., Polish Scientific Publisher PWN (1998), $101-115$.

[107] Th. Schmitt. Some identities for Berezin's function. In Seminar Analysis, 1981/82, pages 146-161. Akad. Wiss. DDR, Berlin, 1982

[108] J. Schwinger. A note on the quantum dynamical principle. Philos. Mag. (7), 44 (1953), $1171-1179$

[109] J. Schwinger. Exterior algebra and the action principle, I. PNAS, 48(4) (1962), 603-611.

[110] I. E. Segal. Tensor algebras over Hilbert spaces. I. Trans. Amer. Math. Soc. 81 (1956), 106-134.

[111] I. E. Segal. Foundations of the theory of dynamical systems of infinitely many degrees of freedom. I. Mat.-Fys. Medd. Danske Vid. Selsk. 31, no. 12, (1959) 39 pp.

[112] J. Sekiguchi. Zonal spherical functions on some symmetric spaces. Publ. Res. Inst. Math. Sci., Kyoto Univ. 12, Suppl., (1977), 455-459.

[113] A. N. Sergeev. Calogero operator and Lie superalgebras. Theoretical and Mathematical Physics 131 (3) (2002), 747-764

[114] D. Shale. Linear symmetries of free boson fields. Trans. Amer. Math. Soc. 103 (1962), 149-167.

[115] D. Shale and W. F. Stinespring. Spinor representations of infinite orthogonal groups. J. Math. Mech. 14 (1965), 315-322.

[116] D. Shklyarov, S. Sinel'shchikov, and L. Vaksman. A q-analogue of the Berezin quantization method, Lett. Math. Phys. 49(3) (1999), 253-261.

[117] W. Soergel. An irreducible not admissible Banach representation of $S L(2, \mathbb{R})$. Proc. Amer. Math. Soc. 104, No.4 (1988), 1322-1324.

[118] A. Unterberger, H. Upmeier. The Berezin transform and invariant differential operators. Comm. Math. Phys. 164 (1994), 563-597.

[119] D.V. Volkov and V.P. Akulov. Possible universal neutrino interaction. JETP Letters 16(11) (1972), 438-440.

[120] D.V. Volkov and V.P. Akulov. Goldstone fields with spin 1/2. Theoretical and Mathematical Physics 18(1) (1974):28-35.

[121] D. V. Volkov and V. A. Soroka. Gauge fields for a symmetry group with spinor parameters. Theoretical and Mathematical Physics 20(3) (1974), 829-834.

[122] Th. Voronov. Geometric Integration Theory on Supermanifolds, volume 9 of Sov. Sci. Rev. C. Math. Phys. Harwood Academic Publ., 1992.

[123] J. Wess and B. Zumino. Supergauge transformations in four dimensions. Nuclear Phys. B 70:39-50, 1974.

[124] J. Wess and B. Zumino. A Lagrangian model invariant under supergauge transformations. Phys. Lett. 49B(1) (1974), 52-54.

[125] A. Weil. Sur certains groupes d'operateurs unitaires. Acta Math. 111 (1964), 143-211.

[126] D. P. Zhelobenko. Operational calculus on a semisimple complex Lie group. Izv. Akad. Nauk SSSR Ser. Mat. 33 (1969), 931-973; English translation in Mat.-USSR-Izvestia 3 (1971), 881-916.

[127] D. P. Zhelobenko and M. A. Naimark. A characterization of completely irreducible representations of a semisimple complex Lie group. Sov. Math. Dokl. 7 (1966), 1403-1406 ; translation from Dokl. Akad. Nauk SSSR 171 (1966), 25-28. 
Department of Mathematics, Abilene Christian University, Box 28012, Abilene, Texas 79699-8012, USA

E-mail address: alexander.karabegov@acu.edu

Institute for Theoretical and Experimental Physics, Bolshaya Cheremushinskaya, 25, 117218 Moscow, Russia

E-mail address: neretin@mccme.ru

School of Mathematics, University of Manchester, Oxford Road, Manchester, M13 9PL, UNiTED KingDOM

E-mail address: theodore.voronov@manchester.ac.uk 